\title{
Amorphous metallic foam
}

\author{
Jan Schroers, ${ }^{\text {a) }}$ Chris Veazey, and William L. Johnson \\ Keck Laboratory of Engineering Materials, California Institute of Technology, Pasadena, California 91125
}

(Received 19 September 2002; accepted 19 November 2002)

The bulk glass forming alloy $\mathrm{Pd}_{43} \mathrm{Ni}_{10} \mathrm{Cu}_{27} \mathrm{P}_{20}$ is processed into a low-density amorphous metallic foam. $\mathrm{Pd}_{43} \mathrm{Ni}_{10} \mathrm{Cu}_{27} \mathrm{P}_{20}$ is mixed with hydrated $\mathrm{B}_{2} \mathrm{O}_{3}$, which releases gas at elevated temperature and/or low pressure. Very homogeneous foams are achieved due to the high viscosity of the alloy even at its liquidus temperature. By processing at the liquidus temperature and decreasing the pressure to $10^{-2}$ mbar, well-distributed bubbles expand to foam the material. Foam densities as low as $1.4 \times 10^{3} \mathrm{~kg} / \mathrm{m}^{3}$ were obtained, corresponding to a bubble volume fraction of $84 \%$. The bubble diameter ranges between $2 \times 10^{-4}$ and $1 \times 10^{-3} \mathrm{~m}$. Thermal analysis by differential scanning calorimetry confirms the amorphous nature of the foam. Furthermore, it reveals that the foam's thermal stability is comparable to the bulk material. (c) 2003 American Institute of Physics.

[DOI: $10.1063 / 1.1537514]$

Metallic foams are known to have many interesting combinations of physical properties. They offer high stiffness in conjunction with very low specific weight, high gas permeability combined with high thermal conductivity, and a very high energy absorption ability. ${ }^{1}$ This combination of properties makes them widely used in nature such as wood or bones. Today, it is an emerging engineering material. Foams can be classified as either open or closed porous. Whereas open foams are mainly used as functional materials, closed foams find application as structural materials such as energy absorbers or light-weight stiff materials. The amount of energy that can be absorbed scales with the strength of the material. Since bulk metallic glasses (BMG) such as $\mathrm{Zr}$ - and Pd-based materials exhibit a yield strength of about $2 \mathrm{GPa},{ }^{2}$ compared to $250 \mathrm{MPa}$ for aluminum, a much larger energy absorption ability can be expected.

The foaming of pure metals is challenging since a foam is an unstable structure. The kinetics of the foam expansion and collapse scales with the viscosity. To a good approximation, the viscosity of pure metals is $10^{-3} \mathrm{Pas}$ at its melting temperature, ${ }^{3}$ compared to about $1 \mathrm{~Pa}$ s for $\mathrm{BMG}$ forming liquids at the liquidus temperature. ${ }^{4}$ Therefore, the foaming kinetics of BMGs can be expected to be more sluggish than that of a pure metal. This should enable better controllability of factors such as foam homogeneity, bubble size distribution, and volume fraction. It has been found that the perfection of a foam strongly influences its mechanical properties. ${ }^{5}$

The challenge of making amorphous foams by cooling the sample from the stable liquid is to circumvent crystallization during subsequent cooling. Thus, techniques used for commercial metal foam production are excluded since they do not allow fast cooling. In order to extract heat sufficiently fast to avoid crystallization, amorphous foam dimensions are restricted in one dimension. In addition, techniques that introduce heterogeneous nucleation for improved bubble distribution will also facilitate crystallization, thereby degrading the glass forming ability. As a foaming material we chose the

\footnotetext{
a) Author to whom correspondence should be addressed; electronic mail: schroers@caltech.edu
}

excellent glass former $\mathrm{Pd}_{43} \mathrm{Ni}_{10} \mathrm{Cu}_{27} \mathrm{P}_{20},{ }^{6}$ which has a critical cooling rate as low as $0.1 \mathrm{~K} / \mathrm{s}$ when processed in $\mathrm{B}_{2} \mathrm{O}_{3}$. Heterogeneous nucleation in this system is actually reduced by processing in $\mathrm{B}_{2} \mathrm{O}_{3} .{ }^{8,9}$

This letter reports on a manufacturing method to foam $\mathrm{Pd}_{43} \mathrm{Ni}_{10} \mathrm{Cu}_{27} \mathrm{P}_{20}$. Hydrated $\mathrm{B}_{2} \mathrm{O}_{3}$ is utilized to create gas bubbles in the liquid melt. These bubbles expand when the pressure is decreased, resulting in a low-density closed-cell foam. Differential scanning calorimetry (DSC) measurements will be carried out to confirm the amorphous nature of the foam.

Ingot material of a purity ranging from $99 \%$ to $99.999 \%$ was used. The prefoam material was prepared by mixing the ingots with $\mathrm{B}_{2} \mathrm{O}_{3}$ powder in quartz tubes, and processing for $1200 \mathrm{~s}$ at $1200 \mathrm{~K}$, followed by water quenching. The temperature was measured with $K$-type thermocouples. Wavelength-dispersive-spectrometer measurements were carried out to verify the composition of the samples. Thermal analysis to confirm the amorphous nature of the foam was performed in a Perkin Elmer DSC 7 analyzer.

Figure 1 shows a prefoam specimen prepared in the de-

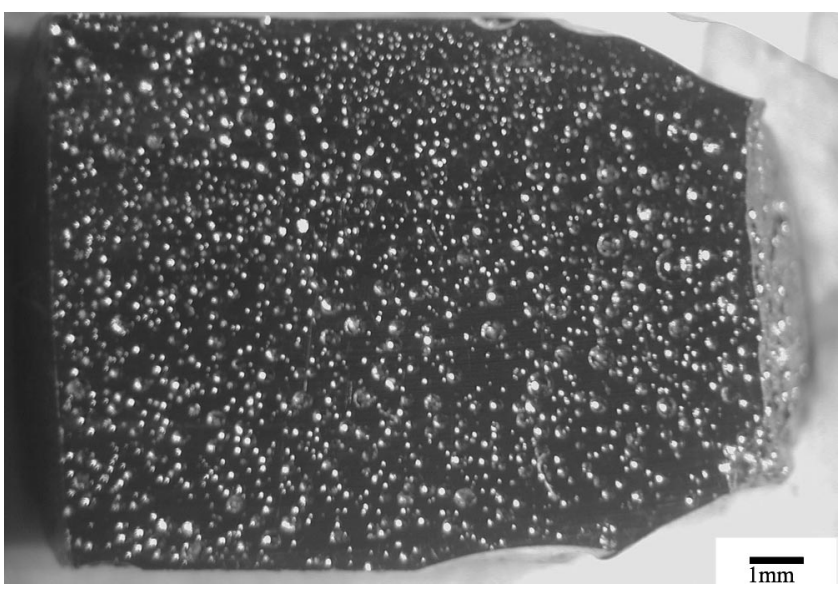

FIG. 1. Prefoam of the amorphous foam prepared by mixing the ingots with $\mathrm{B}_{2} \mathrm{O}_{3}$ and processing for $1200 \mathrm{~s}$ at $1200 \mathrm{~K}$. Bubbles are uniformly distributed throughout the sample. The bubble volume fraction is about $20 \%$ with an average bubble size of $1.5 \times 10^{-3} \mathrm{~m}$. 


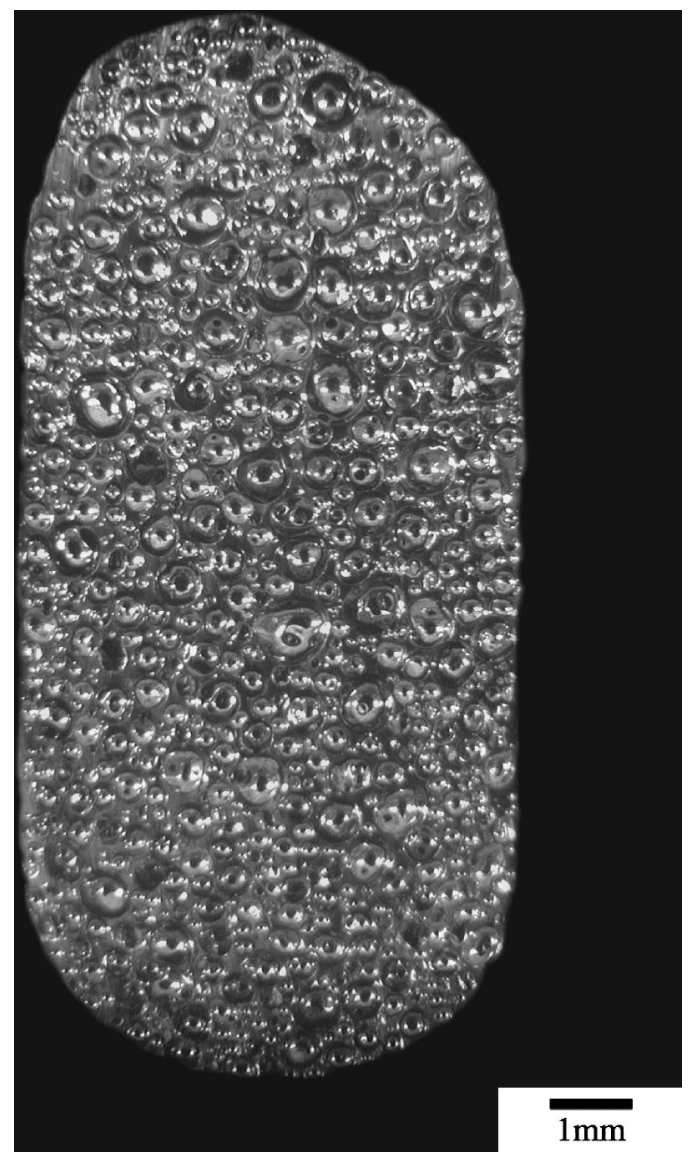

FIG. 2. $\mathrm{Pd}_{43} \mathrm{Ni}_{10} \mathrm{Cu}_{27} \mathrm{P}_{20}$ foam prepared from the prefoam by decreasing the pressure at the liquidus temperature to $10^{-2}$ mbar. The bubbles vary in size between $10^{-4} \mathrm{~m}$ and $10^{-3} \mathrm{~m}$. The density of this foam is 2.2 $\times 10^{3} \mathrm{~kg} / \mathrm{m}^{3}$, which is $24 \%$ of the density of $\mathrm{Pd}_{43} \mathrm{Ni}_{10} \mathrm{Cu}_{27} \mathrm{P}_{20}$.

scribed way. It consists of a uniform distribution of bubbles. The bubble volume fraction is about $20 \%$ with an average bubble size of $1.5 \times 10^{-4} \mathrm{~m}$.

Such a prefoam sample, as is shown in Fig. 1, was processed for $60 \mathrm{~s}$ at $1100 \mathrm{~K}$ to improve the glass forming ability, and subsequently cooled to the alloy's liquidus temperature of about $860 \mathrm{~K}$. Subsequently, the pressure was slowly decreased to about $10^{-2}$ mbar. The lowering of the pressure results in the foaming of the material. After $20 \mathrm{~s}$, when the material reaches its maximum foam height, it was water quenched. The resulting structure is shown in Fig. 2. The density of this foam is $2.2 \times 10^{3} \mathrm{~kg} / \mathrm{m}^{3}$ compared to $9.1 \times 10^{3} \mathrm{~kg} / \mathrm{m}^{3}$ for the bulk $\mathrm{Pd}_{43} \mathrm{Ni}_{10} \mathrm{Cu}_{27} \mathrm{P}_{20}$ sample. ${ }^{10}$ The bubbles vary in diameter between $10^{-4}$ and $10^{-3} \mathrm{~m}$. A very uniform bubble distribution can be observed without noticeable gradient from top to bottom.

A density gradient of the bubbles would establish if the time for the bubble to sediment through the sample is shorter than the experimental time, assuming that the foaming solely results from the growth of pre-existing bubbles and no further nucleation. The sedimentation velocity of a gas bubble in a $\mathrm{Pd}_{43} \mathrm{Ni}_{10} \mathrm{Cu}_{27} \mathrm{P}_{20}$ liquid of viscosity $\eta$ can be calculated according to Stoke's law:

$$
V_{\text {sed }}=2 \quad a^{2}\left(\rho_{l}-\rho_{g}\right) g / 9 \eta,
$$

with gravitational acceleration $g$, bubble radius $a$, and the densities of liquid and gas $\rho_{l}$ and $\rho_{g}$, respectively. The sample the thermogram is shown at the top of Fig. $3, T_{g}$
Downloaded 17 Aug 2007 to 131.215 .225 .175 . Redistribution subject to AlP license or copyright, see http://apl.aip.orglapl/copyright.jsp

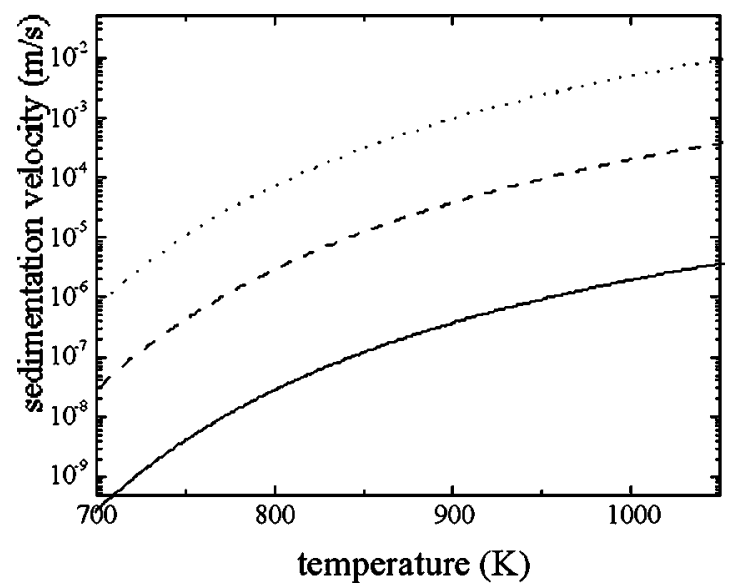

FIG. 3. Mean sedimentation velocity calculated by combining Eqs. (1) and 2 as a function of temperature for a bubble fraction of $30 \%$. Three different bubble sizes of $5 \times 10^{-3} \mathrm{~m}$ (dotted line), $5 \times 10^{-4} \mathrm{~m}$ (dashed line), and 1 $\times 10^{-4} \mathrm{~m}$ (solid line) have been considered.

Stoke's law neglects the hydrodynamic interaction between the bubbles. This interaction, which reduces the sedimentation velocity, is considered in the empirical law of Richardson and Zaki given by

$$
V / V_{\text {sed }}=(1-c)^{n},
$$

with $V$ as the mean sedimentation velocity of the interacting bubbles, $c$ the volume concentration of bubbles, and the empirically determined exponent $n \approx 5$.

Using the viscosity data from Nishiyama and Inoue ${ }^{11}$ and a density of $\rho=9.1 \times 10^{3} \mathrm{~kg} / \mathrm{m}^{3},{ }^{10}$ the sedimentation velocity for gas bubbles in a PdNiCuP liquid as a function of temperature was calculated by combining Eqs. (1) and (2). For simplicity, a constant bubble volume fraction of $30 \%$ was assumed (see Fig. 3). Three different bubble sizes of 5 $\times 10^{-3}, 5 \times 10^{-4}$, and $1 \times 10^{-4} \mathrm{~m}$ have been considered. At a temperature of $900 \mathrm{~K}$ with a corresponding viscosity of 86 $\mathrm{Pas}$, the velocities of $V=1 \times 10^{-3} \mathrm{~m} / \mathrm{s}$ for bubbles with a radius of $5 \times 10^{-3} \mathrm{~m}, V=4 \times 10^{-5} \mathrm{~m} / \mathrm{s}$ for the $5 \times 10^{-4} \mathrm{~m}$ bubble, and $V=4 \times 10^{-7} \mathrm{~m} / \mathrm{s}$ for the $1 \times 10^{-4} \mathrm{~m}$ bubble were calculated. By comparing the time scale for sedimentation with the time scale of the experiment of $20 \mathrm{~s}$, one can conclude that no noticeable gradient should be expected for bubbles with a radius of $5 \times 10^{-4} \mathrm{~m}$ or less. At this point, it should be mentioned that Eq. (1) suggests that the sedimentation velocity of gas bubbles in pure metals is three orders of magnitude higher owing to the three orders of magnitude lower viscosity. Therefore, in liquid pure metals, even bubbles with a radius of $1 \times 10^{-4} \mathrm{~m}$ would show significant sedimentation on the time scale of the experiment.

In order to determine whether the foam can be vitrified upon cooling we performed a thermal analysis measurement by heating the materials with $0.33 \mathrm{~K} / \mathrm{s}$. The thermograms are shown in Fig. 4. Both thermograms for the samples, whose microstructures are shown in Figs. 1 and 2, look very similar. The thermogram for the prefoam sample is shown at the bottom. The glass transition temperature is $T_{g}=578 \mathrm{~K}$, crystallization temperature is $T_{x}=667 \mathrm{~K}$, and the heat of crystallization is $\Delta H=3 \times 10^{3} \mathrm{~J} / \mathrm{mol}$. Solidus and liquidus temperatures are 804 and $859 \mathrm{~K}$, respectively. For the foamed sample the thermogram is shown at the top of Fig. $3, T_{g}$ to AIP license or copyright, see http://apl.aip.org/apl/copyright.js 


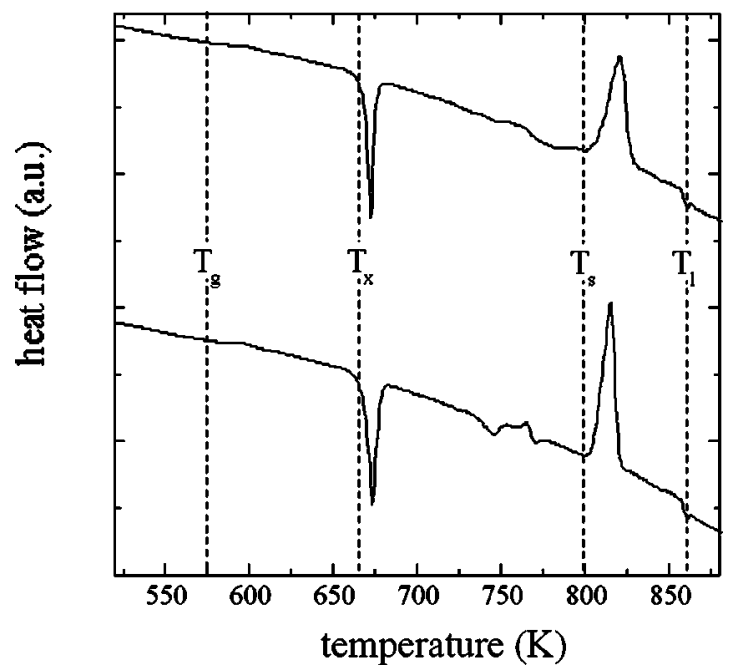

FIG. 4. DSC thermograms of heating experiments with $20 \mathrm{~K} / \mathrm{min}$. The bottom thermogram was obtained for the prefoam material (shown in Fig. 1) and the top thermogram for the foamed material which microstructure is shown in Fig. 2. For the prefoam material $T_{g}=578 \mathrm{~K}, T_{x}=667 \mathrm{~K}, \Delta H$ $=3 \times 10^{3} \mathrm{~J} / \mathrm{mol}, T_{s}=804 \mathrm{~K}$, and $T_{l}=859 \mathrm{~K}$ have been measured. Almost identical values of $T_{g}=577 \mathrm{~K}, T_{x}=666.8 \mathrm{~K}, \Delta H=2.9 \times 10^{3} \mathrm{~J} / \mathrm{mol}, T_{s}$ $=804 \mathrm{~K}$, and $T_{l}=858 \mathrm{~K}$ are determined for the foam.

$=577 \mathrm{~K}, T_{x}=666.8 \mathrm{~K}$, and $\Delta H=2.9 \times 10^{3} \mathrm{~J} / \mathrm{mol}$. Solidus and liquidus temperatures are 804 and $858 \mathrm{~K}$, respectively. The fact that $T_{g}$ and $T_{x}$ are not altered by the foaming process indicates that the foaming does not degrade the glass forming ability. A similar heat of crystallization measured for the two alloys proves that the foam is entirely amorphous.

The influence of the foaming process on the critical casting thickness, assuming the foaming process does not cause heterogeneous nucleation, can be estimated through the increase in thermal diffusion length. If $\alpha_{g} \ll \alpha_{l}(\alpha$ : thermal conductivity), $\rho_{g} \ll \rho_{l}$, and $c_{p, g} \leqslant c_{p, l}$ the heat will predominately transfer through the liquid. But, this requires an increased diffusion length since the linear path is interrupted. Assuming a dense packing of spherically shaped, uniform bubbles, the additional diffusion length can be calculated by comparing the length of going around a bubble with the bubble diameter, resulting in a factor of $\pi / 2$. This results in a critical casting thickness, which is $35 \%$ smaller for a foam compared to the bulk material.

The bulk metallic glass forming alloy $\mathrm{Pd}_{43} \mathrm{Ni}_{10} \mathrm{Cu}_{27} \mathrm{P}_{20}$ was processed into amorphous foam. First, a prefoam was prepared by mixing the ingot materials with $\mathrm{B}_{2} \mathrm{O}_{3}$ powder and processing above the liquidus temperature. The foaming was achieved by decreasing the pressure, which causes preexisting bubbles to grow in the liquid sample. The resulting foams have densities as low as $1.4 \times 10^{3} \mathrm{~kg} / \mathrm{m}^{3}$ with a homogeneous distribution of bubbles of a size range between 2 $\times 10^{-4}$ and $1 \times 10^{-3} \mathrm{~m}$. The homogeneity of the foam is a result of a very low sedimentation velocity, which can be explained by the high viscosity of bulk metallic glass forming liquids.

This work was supported by Liquidmetal Technology. The authors express their gratitude to Kristen Virdone for technical assistance.

${ }^{1}$ J. Banhart, Prog. Mater. Sci. 46, 559 (2002).

${ }^{2}$ H. A. Bruck, T. Christman, A. J. Rosakis, and W. L. Johnson, Scr. Mater. 30, 429 (1994).

${ }^{3}$ T. Iida and R. I. L. Guthrie, The Physical Properties of Liquid Metals (Clarendon, Oxford, U.K., 1988).

${ }^{4}$ A. Masuhr, T. A. Waniuk, R. Busch, and W. L. Johnson, Phys. Rev. Lett. 82, 2290 (1999).

${ }^{5}$ A. E. Evans, J. W. Hutchinson, and M. F. Ashby, Prog. Mater. Sci. 43, 171 (1999).

${ }^{6}$ I.-R. Lu, G. Wilde, G. P. Görler, and R. Willnecker, J. Non-Cryst. Solids 250-252, 577 (1999).

${ }^{7}$ J. Schroers, R. Busch, and W. L. Johnson, Appl. Phys. Lett. 77, 1158 (2000).

${ }^{8}$ H. W. Kui, A. L. Greer, and D. Turnbull, Appl. Phys. Lett. 45, 615 (1984).

${ }^{9}$ J. Schroers, Y. Wu, and W. L. Johnson, Philos. Mag. A 82, 1207 (2002).

${ }^{10}$ I.-R. Lu, G. P. Görler, and R. Willnecker, Appl. Phys. Lett. 80, 4534 (2002).

${ }^{11}$ N. Nishiyama and A. Inoue, Acta Mater. 47, 1487 (1999). 